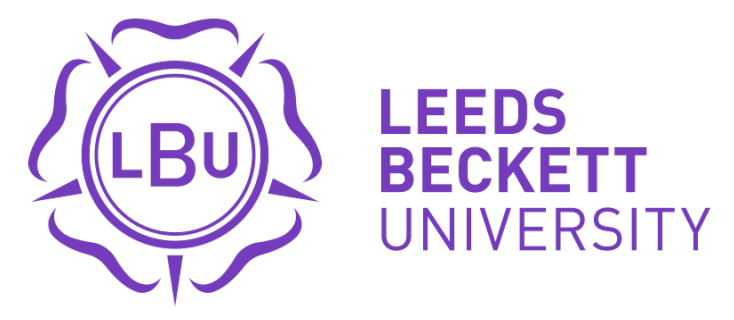

Citation:

Dekker, RL and Morton, $\mathrm{CH}$ and Singleton, PM and Lyndon, A (2016) Women's Experiences Being Diagnosed With Peripartum Cardiomyopathy. Journal of Midwifery and Womens Health. ISSN 1542-2011 DOI: https://doi.org/10.1111/jmwh.12448

Link to Leeds Beckett Repository record:

https://eprints.leedsbeckett.ac.uk/id/eprint/2774/

Document Version:

Article (Accepted Version)

The aim of the Leeds Beckett Repository is to provide open access to our research, as required by funder policies and permitted by publishers and copyright law.

The Leeds Beckett repository holds a wide range of publications, each of which has been checked for copyright and the relevant embargo period has been applied by the Research Services team.

We operate on a standard take-down policy. If you are the author or publisher of an output and you would like it removed from the repository, please contact us and we will investigate on a case-by-case basis.

Each thesis in the repository has been cleared where necessary by the author for third party copyright. If you would like a thesis to be removed from the repository or believe there is an issue with copyright, please contact us on openaccess@leedsbeckett.ac.uk and we will investigate on a case-by-case basis. 


\title{
Women's experiences being diagnosed with peripartum cardiomyopathy: A qualitative study
}

\section{PRECIS}

Despite experiencing severe symptoms, nearly $40 \%$ of women with peripartum cardiomyopathy experienced delays in diagnosis due to symptom dismissal by health care professionals.

\begin{abstract}
Background: Cardiovascular disease has been identified as the leading cause of maternal mortality in the United States, with cardiomyopathy, including peripartum cardiomyopathy (PPCM) accounting for 12-16\% of all pregnancy-related deaths. The purpose of this study was to describe women's experience of being diagnosed with PPCM.
\end{abstract}

Methods: This investigation was conducted using a qualitative design. We collected publicly available narratives posted by 92 women with PPCM ( $29 \pm 6$ years old, ejection fraction $25.5 \% \pm$ 10.8 ) in 3 online support groups. Data were coded and thematically organized so as to produce a richly detailed account of this experience.

Results: The experience of diagnosis was marked by the women's distinct memories of their initial symptoms and whether they were dismissed or taken seriously. The most commonly reported symptoms were extreme shortness of breath, orthopnea, tachycardia, palpitations, chest pain, cough, and edema. Only half of women reported that care providers initially took their symptoms seriously, while a quarter of the women experienced dismissal by health care providers. One-fourth of women were initially given inaccurate diagnoses ranging from "new mom anxiety" to asthma. Women described their initial reaction to diagnosis as feeling terrified, devastated, and feeling a sense of doom. Women had difficulty caring for their newborns during the postpartum period, and they struggled with the medical advice they received to not get pregnant again.

Conclusion: Despite experiencing severe subjective and objective symptoms, about one quarter of women with PPCM experienced symptom dismissal by health care providers, in part due to the overlap between normal symptoms of pregnancy and symptoms of heart failure. 
KEY WORDS: Peripartum cardiomyopathy; heart failure; qualitative; cardiovascular disease; pregnancy

\section{QUICK POINTS}

- Some women with peripartum cardiomyopathy (PPCM) experience a delay in diagnosis due to symptom dismissal by health care professionals

- Women have strong emotional reactions to the diagnosis of PPCM, and they struggle with the advice they receive not to get pregnant again.

- Midwives should be alert to the fact that PPCM is a potential pregnancy and postpartum complication

- Midwives can help women with PPCM understand their prognosis and discuss the dilemma of future childbearing 


\section{Women's experiences being diagnosed with peripartum cardiomyopathy:}

\section{A qualitative study}

This qualitative study aimed to describe women's experience of being diagnosed with peripartum cardiomyopathy (PPCM), defined as the idiopathic development of heart failure and left ventricular systolic dysfunction towards the end of pregnancy or in the months following birth. ${ }^{1}$ Women with PPCM almost always have a reduced left ventricular ejection fraction of less than $45 \%$ (normal values range from 55-65\%). ${ }^{1}$ The incidence of PPCM has risen from 1 in 4,350 live births in 1990-93, to 1 in 2,229 live births in 2000-02, ${ }^{2}$ and is currently 1 in 968 births. ${ }^{3}$ It is not clear whether this increase in PPCM diagnoses is related to an increase in cardiovascular disease risk factors among pregnant women, implementation of the European Society of Cardiology's 2010 expanded diagnostic criteria for PPCM, improved identification of PPCM, or a combination of all three of these factors. ${ }^{3}$

Capturing women's PPCM diagnosis experience is important because cardiovascular disease has been identified as the leading cause of maternal mortality in the United States, with cardiomyopathy, including PPCM, accounting for 12-16\% of all pregnancy-related deaths., ${ }^{4,5}$, Furthermore, delays in care have been identified as contributory to potentially preventable cardiovascular (CVD) deaths. ${ }^{6,7}$ The absolute risk of mortality from PPCM varies, depending on the time period and geographic location in which women are studied. Retrospective studies in the U.S. have revealed absolute PPCM mortality rates ranging from $1.4 \%$ to $18^{\%} .1,7$ One study found an incidence of 3.61 per 10,000 births and a $16.5 \%$ fatality rate over 7 years, with African American women demonstrating a 4-fold higher prevalence and fatality compared with white women. ${ }^{7}$

A diagnosis of PPCM is also associated with significant morbidity, including ventricular dysrhythmias, thromboembolisms and chronic dilated cardiomyopathy. ${ }^{8}$ To make timely and accurate diagnoses, midwives and other health care providers should understand how women identify symptoms and seek medical care, as symptomatology is easily confused with "normal discomforts" of late pregnancy or postpartum. ${ }^{8}$

Our aims were to identify how women describe being diagnosed with PPCM; the symptoms that led them to seek care; the quality of interactions with clinicians during the diagnostic process, and the impact of the diagnosis. Researchers have examined risk factors and long-term health outcomes in mothers with PPCM; however, only one group has reported a 
qualitative study of the experience of living with PPCM, using comments made in a MySpace chat room. ${ }^{9}$ Data on women's experiences with PPCM diagnosis and treatment by health care providers are limited, and so we undertook data analysis of in-depth narratives written by women with PPCM.

\section{METHODS}

\section{Study Design and Sample}

This qualitative study analyzed publicly available narratives posted by women diagnosed with PPCM from 3 websites in mid-2012: "My Heart Sisters," "A Woman's Heart" (no longer online), "PPCMnet" (no longer online). We downloaded 92 narratives and anonymized them prior to analysis. The Institutional Review Board at Stanford University determined this study was not considered human subjects research because data consisted of narratives publicly available on websites.

\section{Data Analysis}

Each narrative file was copied verbatim, given a unique pseudonym, and transferred into the data management software, Dedoose, version 6.2.8 (SocioCultural Research Consultants, LLC, Manhattan Beach, California). We extracted the following data from each narrative: age at diagnosis, parity; year and mode of birth; indication for induction and/or cesarean; complications; and ejection fraction $(\mathrm{EF})$ value at diagnosis and a second time point. Narratives contained limited data in terms of race, marital status, and obstetric history.

Investigators coded and analyzed the text data using thematic analysis according to the methods of Braun and Clarke. ${ }^{10}$ Approaches to thematic analysis are varied, as are criteria for determining what constitutes a "theme" in qualitative research. As Braun and Clarke themselves acknowledge, thematic analysis is not wedded to a particular epistemology and instead benefits from the flexibility needed to provide a rich, detailed and complex account of data in a number of epistemological domains. Our methodological focus was on the examination of themes in experience. The present study aims to make sense of the women's experience by hewing closely to their own narrative structure. Each narrative was read, re-read, and coded. The narratives were compared to ensure that coding was consistent throughout. We deductively coded expected themes such as "identifying symptoms," "seeking medical care," "getting a diagnosis," "treatment and recovery," and "finding meaning." We also sought emergent concepts and 
themes. Examples of codes that emerged from the data analysis include "initial reactions" and "not normal for me."10

We took several steps to encourage rigor, ${ }^{11}$ including designing the study and analyzing data with multiple investigators, meeting frequently throughout data analysis to assure the results reflected the data, and reflexively considering investigator influence in all aspects of the analysis. Analytic triangulation was enhanced by the participation of 2 nurse-researchers, a sociologist, and a social psychologist.

\section{RESULTS}

\section{Description of Sample}

Some women described recent diagnoses, in which prognoses were uncertain. Other narratives were written years after diagnosis, representing the perspectives of long-term survivors. Contextual information provided in the narratives - such as idioms, phrases, language clues, and mention of geographic settings - allowed us to determine that the majority of women who provided narratives likely received care in the U.S. However, it was also clear that from some narratives (7\%), women were from other countries including the United Kingdom, Australia, Canada and the Netherlands.

Table 1 shows demographic and clinical characteristics obtained from the narratives. Not all narratives included this information. About half of women reported being diagnosed after their first pregnancy, while the other half was diagnosed after a subsequent pregnancy. The majority of women $(75.0 \%)$ reported receiving a PPCM diagnosis between 2000 and 2006, while 25.0\% were diagnosed between 2007 and 2012. Of the 69 women who mentioned mode of birth, $72.5 \%$ gave birth by Cesarean, while $27.5 \%$ gave birth vaginally. About $40 \%$ of the sample mentioned experiencing either preeclampsia or hypertension during pregnancy. The average ejection fraction (EF) upon diagnosis was 25.5\% (SD 10.8, range 5\%-54\%), and the average EF upon telling the story was $49.5 \%$ (SD 11.5, range 15\%-75\%).

\section{Diagnosis Experience}

Reports of initial diagnoses of PPCM or congestive heart failure (CHF) were marked by the women's distinct memories of their symptoms, and whether these symptoms were dismissed or taken seriously by themselves, their family/friends, and health care professionals. As women narrated their stories, they referred to the accuracy (or not) of initial diagnoses, and examples of encounters with a variety of health care providers. 
Initial Symptoms. Women described a range of symptoms, including extreme shortness of breath, especially when lying down, very high heart rate, palpitations, chest pressure, cough or asthma unresolved with treatment, excessive edema or swelling, anxiety or restlessness, and significant fatigue or inability to carry out everyday tasks. "My main symptoms were chest pain and sweating. I literally thought I was having a heart attack every second of the day."

Symptom Dismissal. Nearly $40 \%$ of women described how having their symptoms dismissed by physicians and nurses as normal for pregnancy: "Two days after my daughter was born I started to feel as if I couldn't breathe, it was hard work just getting out of bed. I reported this to a nurse who said, 'Of course you're breathing, you haven't turned blue.'”

Women also described not receiving thorough physical exams after reporting symptoms. One woman described how a physician didn't check her swollen ankles - a friend later informed her that she had severe pitting edema. Another woman reported not receiving any physical assessment and being told that her "suffocating" orthopnea was "normal."

Inaccurate Diagnoses. While most women were diagnosed correctly when they initially sought treatment, nearly a third $(n=26)$ reported first being diagnosed with a condition other than PPCM, including: pneumonia, influenza, the common cold, fluid in lungs from magnesium sulfate treatment, gallbladder disease, asthma, "new mom" anxiety, panic attack, stress of having a colicky baby, pulmonary embolus, postpartum depression, and mitral valve regurgitation.

One woman described going to the Emergency Room with severe shortness of breath (SOB) and rapid heartbeat. After 24 hours of being treated for a potential blood clot, new onset asthma, and postpartum depression, she was finally diagnosed with PPCM only after she began to lose consciousness:

I kept begging them to call the doctor and give me something else for the SOB. They offered to call the hospital psychiatrist because they thought I was depressed about not being with my children. After a few hours... when I began to pass out, they rushed me back to ICU. This time my 02 [oxygen saturation] was around 30 and my heart rate was over 200.... I was in critical condition. I just remember passing out about 5 times. They did a bedside electrocardiogram and then determined it must be PPCM. 
When Symptoms Were Taken Seriously. Approximately half of the women (54\%) described having their symptoms taken seriously by a care provider and then being promptly referred to a specialist for cardiac evaluation. However, sometimes women's symptoms were not taken seriously until after they had already been dismissed.

I kept complaining to the nurses I couldn't breathe normally....perhaps they considered me a hysterical new mommy; they gave me Xanax. I stayed awake the entire night; I simply couldn't breathe when I lay down. At seven am, I became frightened. When the nurse (not the one who gave me Xanax, thank God) entered, my oxygen level dropped to 84. I was on my hands and knees, gasping for air. The nurse immediately ran out and I was wheeled to the emergency room and one hour later, I was told I was in congestive heart failure and had PPCM.

Strategies Used to Seek Care. A number of women (20\%) described making multiple visits to seek care and receive a diagnosis, and $18 \%$ sought a second opinion before being diagnosed. Women who had given birth before could compare their symptoms to their first pregnancy, and knew that what they were experiencing was abnormal. However, some firsttime mothers did not have a framework for knowing what was normal or abnormal, and were more hesitant to push for answers. One first-time mother described having an uncomplicated vaginal birth, and noticing 2 months postpartum she was gaining unexplained weight. She began an exercise regimen and thought her symptoms were a result of a too-vigorous workout. Her internist agreed and prescribed an anti-inflammatory medication. A week later she complained of such severe orthopnea and breathlessness that her physician finally ordered an EKG, and she was promptly admitted to the hospital for cardiac evaluation.

Quality of Interactions with Clinicians. Some women assumed clinicians were uncaring when symptoms were not taken seriously: "During all this, I was still trying to breathe. We kept telling everyone something was wrong but they didn't seem to care. I knew that if I stayed there I was going to die." Conversely, women whose diagnoses occurred quickly after onset of symptoms expressed appreciation, especially in contrast to other accounts where the journey to diagnosis took much longer: "I understand reading these other bios that I am extremely lucky to be alive and to have had my condition 
diagnosed so quickly. I am very grateful to all the hospital staff that cared for me during my week in the hospital."

\section{Impact of the Diagnosis}

The second major theme we identified was the impact of the diagnosis. Women described their initial reaction to the diagnosis, coordinating care for a newborn, the impact on future childbearing and relationships, and moving forward with their lives.

Initial Reaction. Women reported experiencing a broad range of emotions in response to the diagnosis, but the most commonly reported were feeling scared, afraid, or terrified, especially in conjunction with an initial pessimistic prognosis:

Some women heard their diagnosis when they were alone. Some also used strong words evoking the depth of their emotions, such as devastating, a sense of doom, hysterical, freaking out and emotional torture:

The cardiologist came in the next day when no one in my family was there, and explained what happened to me and that my heart was functioning at 20\%. He told me that I might need a heart transplant, and that I was finished being pregnant. Of course I was hysterical. I called the nurse in and she could see how upset I was. I think she gave that Dr. a talking to, because a few hours later he came and apologized to me.

A few women had backgrounds in health care, yet even for them, the shock of hearing the diagnosis and prognosis was overwhelming:

Within fifteen minutes of officially being diagnosed I was told by the doctors that I: 1) could have no more children, 2) had to immediately stop breastfeeding, 3) was being admitted to the ICU, and 4) the heart transplant team would be in touch within 24 hours... I was so upset they ended up giving me a sedative.

Managing Care for a Newborn. Between the shock of hearing the diagnosis, and the experience of feeling physically ill, women and their families still had to care for a newborn. This became more complicated when the mother was transferred to another facility or ICU, and 
the baby was well enough to be discharged home. Family members had to choose between supporting the woman and caring for the baby:

I was transferred to a teaching hospital for they were looking into a double transplant option to keep me alive. We have no family in the area and my beautiful healthy babies were at home with my mother. My mother wanted to be with my husband and me. My husband was desperate for help and called the ob/gyn's office for a nurse referral. He left our babies with a complete stranger!

Even having support was problematic, when it reminded women of all they could not do: "I was fortunate to have a sister who had a baby just 5 weeks before me who was able to pump a bottle of breast milk for my baby every day, but that same act of love also depressed me and was a constant reminder that I wasn't well."

Thirteen women wrote about separation concerns - women were in the ICU, and found away from their babies very difficult, at times more difficult than being critically ill. They described how meaningful it was when nurses recognized the value of seeing their baby: "I didn't get to see my son for the first 3 days but then a wonderful nurse let my boyfriend bring him in and all I could do was cry!"

Impact on Childbearing. Nearly half (42 out of 92) of the women were given advice against future childbearing at the same time, or close to, hearing their PPCM diagnosis. All but one of these women were advised against future pregnancies. Only 8 of the 42 women indicated they pursued sterilization to avoid future pregnancies. A few women who weren't planning to have more children were matter-of-fact about their next steps, weighing their current situation against the risks of future childbearing.

Of those who talked about future childbearing, 29 out of 42 were first-time mothers and many struggled to accept the advice to not get pregnant again: "I am very depressed by the fact that my husband and I will not be able to have any more children. This news is completely devastating to me."

Several women ended their narratives with no clear direction as to whether they would try for a future pregnancy; a few noted they received conflicting advice from physicians about the risks of doing so. Women often framed the decision about future childbearing around the 
potential impact of another episode of heart failure on spouses and children: "I was told right from the beginning not to have any more children which upsets me but I could not go through that again, or put my family through that again, maybe even leave my daughter and another child without a mother."

Five women noted that they were in recovery, had achieved normal EFs, and were being weaned off heart medications. These five women were cautiously optimistic about the possibility of another pregnancy. Some asked for women who have given birth successfully after PPCM to share their stories.

Nine women had pregnancies after their PPCM diagnosis. Six were unplanned (one was pregnant at time of posting the narrative) and 5 were monitored in pregnancy and reported good outcomes. Three women planned pregnancies after their PPCM diagnoses: 2 reported uncomplicated pregnancies and good outcomes, and 1 experienced a relapse of PPCM. Women conflicted about having more children stated that reading the stories of women who had successful post-PPCM pregnancies gave them hope.

Impact on Relationships. Several women spoke about the impact of the diagnosis on their marriages and relationships with family members. Some women had families who rallied and pitched in to care for the baby and support the new parents. Yet even with supportive family, some women found themselves forced into solutions that required long periods of separation from their babies so they could focus on their own recovery:

The worst of it was that they put me on a 5 pound lifting restriction, which meant I wasn't even able to pick up my newborn son.... The day before I was released from the hospital, my husband, my parents, and my in-laws got together and decided that I would stay with my parents, my husband would move in with his parents, and my son would be shuffled between my parents' and in-laws' houses every weekend... we didn't feel we had much choice.

Others spoke of the lasting toll on their loved ones:

PPCM stole so much from me and my family. My sister almost didn't get pregnant because of what she saw me go through. She only had one child and had so much anxiety 
through her pregnancy that she didn't have another. That makes me feel guilty.

Sometimes I look at the ripple effect that PPCM caused and I want to take a sword to it and cut it in half...like it did me.

Other women had to go on disability and were concerned that they would not be able to work, and they did not have health insurance or a way to contribute to the household income.

Moving Forward. Most women ended their narratives with a brief summary of their prognosis, and some included expressions of faith and gratitude for their current health status. Several spoke of the mixed blessing of receiving such a diagnosis, with its lifelong implications:

I'm glad that I lived to see Mackenzie's 10th birthday. I'm bitter. I'm thankful. I'm grateful to be alive. I'm pissed. I'm all of these things.

Many women also offered encouragement to anyone who might read their story, and invited others to contact them if they needed someone to talk with about their diagnosis.

\section{DISCUSSION}

One of the key findings of our study is that despite having severe subjective and objective symptoms, one-quarter of women experienced symptom dismissal by health care professionals. When some women presented with complaints of severe breathlessness, orthopnea, palpitations, and even chest pressure, their care providers did not include PPCM among the differential diagnoses, resulting in delayed diagnosis and treatment. Our finding is supported by Hess' et al.'s qualitative analysis of MySpace chat room comments, written by women with PPCM. ${ }^{9}$ Hess et al. found that women used terms such as "dismissed" and "brushed off" when describing seeking treatment for PPCM symptoms. Our study differs from Hess et al.'s in that we included women with diagnoses as recent as 2012 who documented their experiences via in-depth narratives that followed a distinct story-telling pattern, while Hess et al. analyzed PPCM chat room postings from 2005-2008, and some of these postings discussed more than one topic.

In both our study and Hess et al.'s study, some women experienced initial misdiagnoses such as anxiety or normal pregnancy symptoms. Unfortunately, there is considerable overlap between symptoms of PPCM and those of normal pregnancy, which can contribute to treatment delay. Dyspnea, dizziness, edema, and orthopnea can occur in normal pregnancies, ${ }^{12}$ and up to 
$80 \%$ of pregnant women experience peripheral edema. ${ }^{13}$ In a recent study describing cardiovascular disease-related maternal deaths in California between the years 2002-2006, researchers estimated that 13 of the 29 dilated cardiomyopathy maternal deaths (45\%) were due directly to health care providers' failure to respond promptly to warning signs in the patient's history and clinical exam, leading to a delay in diagnosis. ${ }^{7}$

To help reduce symptom dismissal and improve the outcomes of women with PPCM, it is important for midwives, physicians, and nurses who work with pregnant and postpartum women to be aware of PPCM symptoms and keep this rare but serious differential diagnosis in mind when working with a mothers who have potential cardiac-related complaints. Women who are age 40 or older, obese, African American, or have a history of diabetes, hypertension, or substance abuse, are at additional risk factors for developing PPCM. However, regardless of the presence of risk factors for PPCM, midwives should include a cardiac etiology in their differential diagnosis if any of the following red flags are present on physical exam: shortness of breath at rest, orthopnea, resting heart rate $>120$ beats per minute, resting systolic blood pressure $>160 \mathrm{~mm} \mathrm{Hg}$, resting respiratory rate of > 30 breaths per minute and an oxygen saturation < $94 \% .{ }^{6}$ The presence of any red flags indicates a need for prompt evaluation and referral to a primary care physician, maternal-fetal medicine specialist, or cardiologist. While waiting for the referral, midwives can immediately order an electrocardiogram (EKG) and a B-type natriuretic peptide (BNP) laboratory test. The BNP is a simple, inexpensive screening test that - if elevated - is highly specific for left ventricular systolic dysfunction. According to the California Maternal Quality Care Collaborative's soon-to-be-released toolkit on PPCM, a positive result on a BNP or EKG indicates a high suspicion for cardiovascular disease. ${ }^{14}$

In our study, we found that women had strong emotional reactions upon learning their PPCM diagnosis. Our finding is consistent with Hess's qualitative study, ${ }^{9}$ in which women with PPCM used terms such as "shocked" and terrified" when they described receiving their diagnosis. The diagnosis of PPCM needs to be given in a sensitive manner, in the presence of the woman's support system. Women also need accurate, evidence-based information about their risk of mortality and prognosis for recovery. Although women in our study found the diagnosis of PPCM to be a terrifying experience, actual survival rates are high. In 2000, Felker et al. ${ }^{15}$ found that women with PPCM have a 94\% survival rate at 5 years. The high survival rate is possibly due to the younger age and less comorbidity at diagnosis in comparison to patients 
diagnosed with heart failure due to other causes. ${ }^{12,15}$ In one registry study, $78 \%$ of mothers with PPCM regained normal systolic function, and all women with an EF of $35 \%$ or higher at the time of diagnosis eventually recovered. ${ }^{16}$

Consistent with Hess et al.'s study, ${ }^{9}$ many women in our study voiced concerns about the impact of PPCM on their future childbearing potential. Evidence shows that approximately onethird of women who get pregnant will experience a relapse. ${ }^{17}$ The risk of experiencing PPCM is related to a woman's current cardiac function and is thus individualized. ${ }^{17}$ Therefore, women with PPCM may need ongoing counseling as to the pros and cons of future childbearing, types of birth control available, potential for relapse should they become pregnant, and the importance of avoiding pregnancy while they still have a reduced EF. ${ }^{18}$

Patient stories are increasingly recognized as central to medical care, ${ }^{19}$ and our findings suggest PPCM narratives generate rich data. More clinical research on PPCM is needed, using mixed-methods or qualitative components to capture women's experiences and integrate them with treatment and outcome data, to achieve truly patient-centered recommendations for care. However, the fact that two groups of qualitative researchers have now documented similar themes from women with PPCM- experiencing dismissal by care providers, feeling terrified during the diagnosis-telling, and being distressed about future pregnancies - makes it clear that clinicians can take action to provide better emotional and informational support for women with this rare diagnosis. The results from our study reflect the importance of listening to a woman's complaints of severe symptoms, and valuing women's knowledge of what is normal and abnormal for them.

\section{Limitations}

Our study was limited by its collection of pre-existing narratives posted prior to 2012 on peer support websites focused on peripartum cardiomyopathy, without the opportunity to collect full demographic data and obstetric histories, or to ask women further about their experiences. The inability to explore the written narratives further with women truncates researchers' ability to clarify meaning and range of variation in the data. Furthermore, the use of internet narratives limits the sample to persons with internet access and effective written communication skills. However, given that PPCM is a rare diagnosis, this method allowed us to analyze findings from a much larger sample than would have been practical in a traditional study. 
Women in our study were at differing points in the diagnosis journey - rarely in the midst of the crisis, but instead months or years later. Similarly, the diagnosis experience occurred in diverse settings over a wide range of time. Despite these limitations, the large number of narratives and the recurrent themes represent a rich perspective on women's experiences receiving a PPCM diagnosis. We believe that future investigators who conduct research on women with PPCM should include a mixed-methods approach, collecting women's experiences as well as assessing their emotional and mental health response to the PPCM diagnosis.

\section{CONCLUSIONS}

Despite experiencing severe subjective and objective symptoms, nearly $40 \%$ of women with PPCM experienced symptom dismissal by health care providers, partially due to the overlap between normal pregnancy symptoms and symptoms of heart failure. Clinicians who work with women of childbearing age should be knowledgeable about PPCM symptoms and keep this rare but serious differential diagnosis in mind when encountering women who experience severe edema, dyspnea, orthopnea, or tachycardia. Health care providers can help women diagnosed with PPCM to understand their prognosis and how PPCM will impact future childbearing. 


\section{REFERENCES}

1. Sliwa K, Hilfiker-Kleiner D, Petrie MC, et al. Current state of knowledge on aetiology, diagnosis, management, and therapy of peripartum cardiomyopathy: a position statement from the Heart Failure Association of the European Society of Cardiology working group on peripartum cardiomyopathy. Eur J Heart Fail. 2010;12(8):767-778.

2. Mielniczuk LM, Williams K, Davis DR, et al. Frequency of peripartum cardiomyopathy. Am J Cardiol. 2006;97(12):1765-1768.

3. Kolte D, Khera S, Aronow WS, et al. Temporal trends in incidence and outcomes of peripartum cardiomyopathy in the United States: a nationwide population-based study. $J$ Am Heart Assoc. 2014;3(3):e001056.

4. Creanga AA, Berg CJ, Syverson C, Seed K, Bruce FC, Callaghan WM. Pregnancyrelated mortality in the United States, 2006-2010. Obstet Gynecol. 2015;125(1):5-12.

5. Main EK, McCain CL, Morton CH, Holtby S, Lawton ES. Pregnancy-related mortality in California: causes, characteristics, and improvement opportunities. Obstet Gynecol. 2015;125(4):938-947.

6. Hameed A, Lawton E, McCain C, et al. Pregnancy-related cardiovascular deaths in California: Beyond peripartum cardiomyopathy. [published online ahead of print May 13, 2015]. Am J Obstet Gynecol. doi: 10.1016/j.ajog.2015.05.008.

7. Harper MA, Meyer RE, Berg CJ. Peripartum cardiomyopathy: population-based birth prevalence and 7-year mortality. Obst Gynecol. 2012;120(5):1013-1019.

8. Fett JD. Earlier detection can help avoid many serious complications of peripartum cardiomyopathy. Future Cardiol. 2013;9(6):809-816.

9. Hess RF, Weinland JA. The life-changing impact of peripartum cardiomyopathy: an analysis of online postings. MCN Am J Matern Child Nurs. 2012;37(4):241-246.

10. Braun V, Clarke V. Using thematic analysis in psychology. Qual Res Psychol. 2006;3:77101.

11. Tracy SJ. Qualitative quality: eight "big-tent" criteria for excellent qualitative research. Qualitative Inquiry. 2010;16: 837-851.

12. Ramaraj R, Sorrell VL. Peripartum cardiomyopathy: causes, diagnosis, and treatment. Cleve Clin J Med. 2009;76(5):289-296. 
13. Cho S, Atwood JE. Peripheral edema. Am J Med. 2002;113(7):580-586.

14. Hameed AB, Morton $\mathrm{CH}$, Moore A. Cardiovascular disease in pregnancy and postpartum toolkit: improving health care response to cardiovascular disease in pregnancy and postpartum. Developed under contract \#11-10006 with the California Department of Public Health; Maternal, Child, and Adolescent Health Division: California Maternal Quality Care Collaborative; 2015.

15. Felker GM, Jaeger CJ, Klodas E, et al. Myocarditis and long-term survival in peripartum cardiomyopathy. Am Heart J. 2000;140(5):785-791.

16. Safirstein JG, Ro AS, Grandhi S, Wang L, Fett JD, Staniloae C. Predictors of left ventricular recovery in a cohort of peripartum cardiomyopathy patients recruited via the internet. Int J Cardiol. 2012;154(1):27-31.

17. Elkayam U. Risk of subsequent pregnancy in women with a history of peripartum cardiomyopathy. J Am Coll Cardiol. 2014;64(15):1629-1636.

18. Fett JD, Shah TP, McNamara DM. Why do some recovered peripartum cardiomyopathy mothers experience heart failure with a subsequent pregnancy? Curr Treat Options Cardiovasc Med. 2015;17(1):354.

19. Berwick DM. The stories beneath. Med Care. 2007;45(12):1123-1125. 
Table 1: Reported demographic and clinical characteristics from women's PPCM narratives ${ }^{\mathrm{a}}(\mathrm{N}$ =92)

\begin{tabular}{|c|c|c|}
\hline $\begin{array}{l}\text { Demographic and Clinical } \\
\text { Characteristics }\end{array}$ & $\begin{array}{c}\text { Number Reporting } \\
(\%)\end{array}$ & $\begin{array}{c}\text { Mean } \pm \text { standard } \\
\text { deviation }\end{array}$ \\
\hline Age at diagnosis & $\begin{array}{c}50(54.3) \\
\text { Range 17- } 41\end{array}$ & $29.1(6)$ \\
\hline Parity & 91 & -- \\
\hline First pregnancy & $49(50.5)$ & -- \\
\hline Second pregnancy & $24(24.7)$ & -- \\
\hline Third pregnancy & $10(10.3)$ & -- \\
\hline Fourth or higher & $8(8.2)$ & -- \\
\hline Unreported & 1 & -- \\
\hline Year of diagnosis & $76(82.6)$ & -- \\
\hline Prior to 2000 & $14(18.4)$ & -- \\
\hline $2000-2006$ & $43(56.6)$ & -- \\
\hline $2007-2010$ & $19(25.0)$ & -- \\
\hline Unreported & 16 & -- \\
\hline Mode of birth & $69(75.0)$ & -- \\
\hline Vaginal & $19(27.5)$ & -- \\
\hline Cesarean & $50(72.5)$ & -- \\
\hline Unreported & 23 & -- \\
\hline Indication for Cesarean $^{\text {b }}$ & $44(47.8)$ & -- \\
\hline Failed induction & $17(38.6)$ & -- \\
\hline Maternal condition & $11(25.0)$ & -- \\
\hline Malposition & $5(11.4)$ & -- \\
\hline Fetal distress & $5(11.4)$ & -- \\
\hline Failure to progress & $2(.05)$ & -- \\
\hline Scheduled or repeat & $2(.05)$ & -- \\
\hline Twin birth & $2(0.5)$ & -- \\
\hline Unreported & 6 & -- \\
\hline
\end{tabular}




\begin{tabular}{|c|c|c|}
\hline $\begin{array}{l}\text { Demographic and Clinical } \\
\text { Characteristics }\end{array}$ & $\begin{array}{c}\text { Number Reporting } \\
(\%)\end{array}$ & $\begin{array}{l}\text { Mean } \pm \text { standard } \\
\text { deviation }\end{array}$ \\
\hline Initial diagnosis & $87(94.6 \%)$ & -- \\
\hline $\begin{array}{l}\text { Peripartum cardiomyopathy or } \\
\text { congestive heart failure }\end{array}$ & $68(77.3)$ & -- \\
\hline Pneumonia & $8(9.1)$ & -- \\
\hline Pulmonary edema & $2(2.3)$ & -- \\
\hline Preeclampsia & $4(4.5)$ & -- \\
\hline Hypertension & $1(1.1)$ & -- \\
\hline Asthma & $2(2.3)$ & -- \\
\hline Postpartum depression & $2(2.3)$ & -- \\
\hline $\begin{array}{l}\text { Preeclampsia or hypertension during } \\
\text { pregnancy }\end{array}$ & & -- \\
\hline Preeclampsia & $16(16.5)$ & -- \\
\hline Hypertension & $21(21.6)$ & -- \\
\hline $\begin{array}{l}\text { No mention of preeclampsia or } \\
\text { hypertension }\end{array}$ & $55(59.8)$ & -- \\
\hline Initial ejection fraction & 77 & $\begin{array}{c}25.5(10.8) \\
\text { Range 5-54\% }\end{array}$ \\
\hline Most recent ejection fraction & 72 & $\begin{array}{c}49.5(11.5) \\
\text { Range } 15-75 \%\end{array}$ \\
\hline
\end{tabular}

${ }^{a}$ Not all narratives contained all demographic and clinical characteristics compiled in this table.

${ }^{b}$ Indications for Cesarean were not always explicitly stated by the women and the categories here were assigned by the researchers. 